Revue d'histoire de l'Amérique française

REVUE D.HISTOIRE DE L'AMÉRIQUE FRANÇAISE

\title{
Inventaire général des Dossiers des archives de la chancellerie de l’Archevêché de Montréal, de l'année 1600 à l'année 1876
}

\section{François Beaudin}

Volume 19, numéro 4, mars 1966

URI : https://id.erudit.org/iderudit/302534ar

DOI : https://doi.org/10.7202/302534ar

Aller au sommaire du numéro

Éditeur(s)

Institut d'histoire de l'Amérique française

ISSN

0035-2357 (imprimé)

1492-1383 (numérique)

Découvrir la revue

Citer ce document

Beaudin, F. (1966). Inventaire général des Dossiers des archives de la chancellerie de l'Archevêché de Montréal, de l'année 1600 à l'année 1876. Revue d'histoire de l'Amérique française, 19(4), 652-664.

https://doi.org/10.7202/302534ar d'utilisation que vous pouvez consulter en ligne. 


\section{INVENTAIRE D'ARCHIVES}

\section{INVENTAIRE GÉNÉRAL DES DOSSIERS \\ DES ARCHIVES DE LA CHANCELLERIE DE L'ARCHEVÊCHÉ DE MONTRÉAL DE L'ANNÉE 1600 À L'ANNÊE 1876}

\section{ARCHIVES DE LA CHANCELLERIE DE L'ARCHEVÊCHÊ DE MONTREAL (ACAM)}

$\mathrm{Au}$ cours de son congrès annuel de juin 1963, la Société canadienne d'Histoire de l'Église catholique mettait à son programme une conférence du Père Gaston Carrière, o.m.i., qu'il intitulait: "Les richesses inexploitées de nos archives diocésaines". ${ }^{1}$

Le Père Carrière notait alors combien le travail qui restait à accomplir dans le domaine des archives diocésaines était vaste comparé à ce qui avait déjà été fait.

C'est dans cet esprit de mise en valeur des archives diocésaines de Montréal que Son Éminence le Cardinal Paul-Émile Léger, Archevêque de Montréal, le 9 mai 1963, nommait un titulaire à temps plein aux Archives de la Chancellerie de l'Archevêché de Montréal, sa nomination devenant effective le 15 juin suivant.

On trouvera dans La Société canadienne d'Histoire de l'Église catholique - Rapport 1964, aux pp. 11 à 14 incl., un "Bref aperçu sur l'Histoire des Archives de la Chancellerie de l'Archevêché de Montréal". Ce court article permettra de se familiariser avec ce dépôt d'archives et de connaître les étapes de sa formation.

${ }^{1}$ Cf. Revue de l'Univ. d'Ottawa, vol. 34 (1964): 7396, et La Société canadienne d'Histoire de l'Eblise catholique - Rapport 1963: 63-81. 
Dans la revue bibliographique qu'il consacrait ici à cet aperçu $^{2}, M$. Cameron Nish déplorait que ce dernier traitât de "l'Administration des Archives plutôt que des documents qu'elles renferment".

Le travail de classement, de classification et d'inventaire, et l'organisation du département des Archives de la Chancellerie de l'Archevêché de Montréal (dont le personnel est passé de 1 à 9 personnes) qui ont été réalisés depuis 1963 permettent maintenant de faire connaître aux chercheurs le contenu de ces archives, au moins de façon sommaire.

Pour ce qui concerne la consultation de ces archives, il est important de connaître les renseignements suivants.

Les Archives de la Chancellerie de l'Archevêché de Montréal ne sont pas publiques. Les personnes autres que les membres de la Curie diocésaine doivent, pour pouvoir les consulter, en obtenir l'autorisation. Ces personnes doivent offrir les garanties nécessaires de probité et de sérieux. La demande de consultation est toujours un engagement à se conformer aux règlements établis. La permission de consulter les archives doit être demandée par écrit. À sa demande, on doit joindre une recommandation particulière de la part d'une autorité qui sera, de préférence, religieuse. Si l'on n'est pas citoyen canadien, on doit pouvoir présenter, en plus, son passeport ou quelque autre pièce d'identité visée par l'autorité consulaire locale. On adresse sa demande à: Monsieur le Chancelier, La Chancellerie, 2000 ouest, rue Sherbrooke, Montréal 25. Le département des Archives est ouvert du lundi au vendredi, de 10 hres a.m. à 12 hres, et de 2 hres p.m. à 4.30 hres p.m. Il est à remarquer qu'on ne peut avoir accès pour consultation à des documents reçus ou enregistrés après le mois de septembre 1876 , moment de la démission de $\mathrm{M}^{\mathrm{gr}}$ Ignace Bourget comme évêque de Montréal. Pour renseignements supplémentaires, on peut s'adresser à : L'Archiviste, Archives de la Chancellerie, Archevêché de Montréal, 2000 ouest, rue Sherbrooke, Montréal 25. Téléphone: 935-5481, poste 47.

2 RHAF, XIX (1965-1966) : 310-311. 
Le dépôt des Archives de la Chancellerie de l'Archevêché de Montréal se divise, selon la nature des documents, en quatre secteurs: registres, dossiers contenant des pièces détachées, photos et cartes géographiques ou plans.

Pour ce qui est du secteur "registres", on trouvera dans la conférence du Père Carrière mentionné ci-haut quelques indications. Elles ne peuvent malheureusement donner qu'une faible idée de la richesse de notre dépôt en ce domaine. On y reviendra, espérons-le, dans un article subséquent. Contentons-nous, en attendant, de signaler que les Registres des Lettres et les Registres de la Chancellerie font l'objet d'un Inventaire analytique, qui paraît depuis 1941, dans le Rapport de l'Archiviste du Québec. Momentanément arrêtée de 1957 à 1964, la publication de cet Inventaire a repris dans le tome 42 du Rapport de l'Archi-

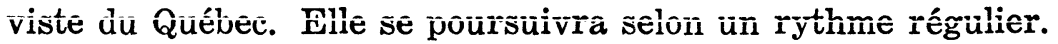

C'est le secteur "dossiers" qui sera ici l'objet de notre attention. On trouvera plus bas un Inventaire général des dossiers des Archives de la Chancellerie de l'Archevêché de Montréal pour les années 1600 à 1876 . Cet Inventaire sera présenté en trois sections selon des morcellements chronologiques à l'intérieur de ces années. Une première période couvrira les années 1600 à 1760. La deuxième période s'étendra de l'année 1760 à l'année 1835 (année précédant l'érection du diocèse de Montréal). Un article subséquent fera connaître l'Inventaire des dossiers contenant des documents datant de la période allant de 1836 à 1876 (année de la démission de $M^{\text {gr }}$ Ignace Bourget comme évêque de Montréal et date limite de consultation).

On se demandera peut-être d'où viennent les documents contenus dans les dossiers des deux premières périodes de cet Inventaire. Voici comment s'est constituée cette partie de notre dépôt. Une partie des documents contenus dans ces dossiers provient de l'administration de $\mathrm{M}^{\mathrm{gr}} \mathrm{J}$.-J. Lartigue comme évêque auxiliaire, vicaire général et suffragant de l'évêque de Québec, durant la période allant de 1821 à 1836. D'autres documents sont des papiers personnels de $\mathrm{M}^{\mathrm{gr}}$ Lartigue. D'autres ont été 
recueillis après la fondation du diocèse en 1836. Le reste vient de l'Archevêché de Québec: obéissant aux lois ecclésiastiques, l'évêque de Québec, au moment de l'érection du diocèse de Montréal, versa aux Archives de celui-ci, en autant que cela était possible, les documents concernant le territoire du nouveau diocèse.

INVENTAIRE GÉNÉRAL DES DOSSIERS

DES ARCHIVES DE LA CHANCELLERIE DE

L'ARCHEVECHE DE MONTREAL

pour les années allant de l'an 1600 à 1876

lère période: $1600-1760$

2ème période: 1760-1835

3ème période: 1836-1876

Voici quelques notes techniques. Par dossiers, il faut entendre un même sujet ou une même institution dont les documents sont groupés ensemble. Selon la quantité de documents, il peut s'agir d'une seule chemise (contenant un seul ou plusieurs documents) ou de plusieurs chemises de classeur métallique.

Pour consultation efficace et rapide, on fera toujours mention de la cote de classification indiquée au début de la ligne.

Les années entre parenthèses au bout de la ligne indiquent l'année du document s'il est unique ou s'ils sont tous de la même année ou bien l'année du plus ancien et du plus récent document contenu dans le dossier mentionné, à l'intérieur cependant des limites extrêmes de la période de l'Inventaire que l'on consulte.

L'astérisque avant la parenthèse de la fin de la ligne indique que ce dossier est aussi mentionné à la période précédente de l'Inventaire. Lorsque l'astérisque se trouve après la parenthèse, cela indique qu'il se continue dans la période qui suit de l'Inventaire. 
I N V E N T A I R E

DES DOSSIERS

CONTENANT DES DOCUMENTS

DE L A

PREMIERE PERIODE

$1600-1760$

P A P E S

1ère Période: $1600-1760$

111.099 Clément VIII (1592-1605)

(1604)

111.104 Innocent $\mathrm{X} \quad$ (1644-1655)

111.116 Clément XIII (1758-1769)

D I O C E S E

295.101 Québec (diocèse de -)

$(1675-1760)^{*}$

P A R O I S E S

355.104 Purification-de-la-B.V.M.

$(1707-1760)^{*}$

355.106 St-François-de-Sales

$(1685-1760)^{*}$

355.113 St-Joseph (Riv.-des-Praries)

$(1759-1760)^{*}$

355.114 L'Assomption

$(1724-1760)^{*}$

355.117 St-Vincent-de-Paul (Laval)

RELIGIEUX PERES

465.103 Société de Jésus (Jésuites)

$(1633-1663)$

R E L I G IE U S E S

525.101 Congrégation Notre-Dame

$(1698-1760)^{*}$

(Dames de la -)

525.102 Hospitalières de St-Joseph (Rel. -) (1676-1760)* 525.103 Sœurs Grises de Montréal

$(1694-1760)^{*}$ 
FONDS LARTIGUE - BOURGET

901.001 Évêques de Québec

(1744-1758)

901.003 Évêques de Québec

(1728-1735)

901.008 Vœu des Hurons

901.011 Pères Récollets

901.028 $\mathrm{M}^{\mathrm{gr}}$ Lartigue - Lettres de M. Montgolfier, Lettres de prêtres et d'amis, Règlements divers et Documents

901.136 Notre-Dame - Division de la Paroisse

$(1709-1760)^{*}$

901.137 Notre-Dame et Saint-Sulpice 901.148 Règlements au sujet des Registres, Cas réservés, etc... (1678-1760)* (1678-1760)* $(1727-1760)^{*}$

I N VENTA IR E

DES DOSSIERS

CONTENANT DES DOCUMENTS

D E L A

DEUXIEME PERIODE

$1760-1835$

2ème Période: 1760-1835

P A P E S

$\begin{array}{llrl}111.120 & \text { Léon XII } & (1823-1829) & (1824-1825) \\ 111.121 & \text { Pie VIII } & (1829-1830) & (1824-1829) \\ 111.122 & \text { Grégoire XVI } & (1831-1846) & (1832-1834)\end{array}$

I N D U L T S

$\begin{array}{cl}149.995 & \begin{array}{l}\text { Index (Indults) } \\ \text { Indults }\end{array} \\ 149.997 & \text { Rescrits (Rome) }\end{array}$

(1806-1835)*

(1806-1835)*

(1800-1835) * 
D I O C E S E S

$\begin{array}{ll}195.103 & \text { Boston } \\ 195.104 & \text { Louisville } \\ 195.105 & \text { New-York } \\ 195.112 & \text { St. Louis } \\ 195.125 & \text { Albany } \\ 255.101 & \text { Terreneuve } \\ 255.102 & \text { Kingston } \\ 255.109 & \text { St-Boniface } \\ 255.110 & \text { Ottawa } \\ 295.101 & \text { Québec } \\ 295.103 & \text { St-Hyacinthe } \\ 295.104 & \text { Trois-Rivières }\end{array}$

(1832-1835)* (1830-1835)* $(1832-1835) *$ (1831-1835)* (1834-1835)* $(1822-1835) *$ (1822-1835)* (1821-1835)* $(1828-1835)^{*}$ * $(1760-1835) *$ $(1825-1835)^{*}$ $(1805-1835)^{*}$

CAISSES ECCLESIASTIQUES

324.201 Caisse ecciésiastique Sti-ivichei (diocèse de Québec) 324.202 Caisse ecclésiastique de Montréal (1834-1835)*

P A R OIS SES

355.102 St-Enfant-Jésus (Pointe-auxTrembles)

$(1796-1835) *$

\begin{tabular}{ll} 
& \multicolumn{1}{c}{ Trembles) } \\
355.103 & SS-Anges de Lachine \\
355.104 & Purification-de-la-B.V.M. \\
355.105 & St-Laurent \\
355.106 & St-François-de-Sales \\
355.107 & St-François-d'Assise \\
355.108 & St-Sulpice \\
355.109 & Ste-Anne-de-Bellevue \\
355.110 & St-Joachim (Pointe-Claire) \\
355.111 & Ste-Geneviève \\
355.112 & Visitation-de-la-B.V.M. \\
355.113 & St-Joseph (Rivière-des-Prairies) \\
355.114 & L'Assomption \\
355.116 & St-Martin \\
355.117 & St-Vincent-de-Paul (Laval)
\end{tabular}

(1787-1835)* $(1768-1835) *$

*(1760-1835)* $(1797-1835)^{*}$ * (1760-1835)* $(1795-1835)^{*}$ $(1772-1835)^{*}$ $(1792-1835) *$ $(1787-1835)^{*}$ $(1778-1835)^{*}$ (1794-1835)*

*(1760-1835) *

* (1760-1835) * (1770-1835)*

*(1760-1835)* 
355.118 Ste-Rose

355.159 Basilique-Cathédrale

C LER GE
(1761-1835)*

$(1822-1835) *$

410.002 Titres Cléricaux

$(1821-1835)^{*}$

Prêtres séculiers du diocèse de Montréal ordonnés avant 1836 et dont des documents ont été repérés (ces documents peuvent être datés avant ou après 1836).

420.019 Plinguet, Vincent. (ordonné le 21.9.1833)

420.029 Brassard, Thomas-Léandre. (ordonné le 5.9.1830)

420.041 Blanchet, Augustin-Magloire. (ordonné le 3.6.1821)

420.045 Porlier, F.P. (ordonné le 21.11.1824)

420.046 Poirier, Isidore. (ordonné le 12.10.1817)

420.048 Chartier, Étienne. (ordonné le 28.12.1828)

420.051 Paquin, Jacques.

(ordonné le 24.9.1814)

420.066 Marcoux, Joseph.

(ordonné le 12.6.1813)

420.093 Charland, Louis-Davis.

(ordonné le 6.9.1835)

420.095 Lafrance, Pierre.

(ordonné le 1.10.1826)

Prêtres étrangers au diocèse de Montréal

$\begin{array}{ccc}450.902 & \text { "B" (Berthelot, Charles) } & (1830)^{*} \\ 450.904 & \text { "D" (Desjardins, L.-J. } \\ & \text { Dolan, Guillaume } \\ & \text { Duval, Joseph } \\ \text { Dugat, ..) } & (1803-1835) * \\ 450.908 \text { "H" (Harper, Jean) } & (1827)^{*}\end{array}$




450.912 “L” (L'Heureux, François)
450.913 “M" (McReavy, Denis)
450.915 “O” (O'Connell, Pierre)
450.916 “P” (Parant, Antoine)
450.918 “R” (Ryder, Rodrigue)

Religieux Pères

465.101 Compagnie de Saint-Sulpice
(1835)*

$(1834-1835)^{*}$ (1832)* $(1833-1834)^{*}$ (1831)*

* (1786-1835) *

\section{RELIGIEUX LAICS}

\section{Sours}

\subsection{Congrégation Notre-Dame}

(Dames de la -)

*(1760-1835)*

525.102 Hospitalières de St-Joseph

$$
\text { (Rel. -) }
$$

52ิ5.10̂s S̃œurs Grises de Múñtréál

525.106 Sœurs de la Providence

L A I C S

$$
\begin{array}{cc}
572.000 & \text { “B" (Boucherville, Thomas) } \\
574.000 & \text { "D" (Delisle, J.-G. } \\
& \text { DeSève, Charles } \\
& \text { Dézery, J.-Bte } \\
& \text { Drolet, Alex.) } \\
576.000 & \text { "F" (Foucher, M.) } \\
577.000 & \text { "G" (Gaume, .. ) } \\
578.000 & \text { "H" (Hamel, Josephte } \\
& \text { Hébert, J.-Bte) } \\
582.000 & \text { "L" (LaFontaine, L.-H.) } \\
583.000 & \text { "M" (Mauduit, M.A., Mlle) } \\
586.000 & \text { "P" (Prévost, J.-L.) } \\
589.000 & \text { "S" (Sauvageau, A.) }
\end{array}
$$

FONDS LARTIGUE-BOUR GET 901.002 Évêques de Québec 
901.004 Étienne Marchand, vic. gén. et curé de Boucherville

901.005 Étienne Montgolfier, vic. gén.

901.006 Charles-Madelaine Dufrost, vic. gén. et curé de Boucherville

901.010 Jean-Jacques Lartigue, év. de Mtl, (diverses nominations)

(1766-1771)

(1760-1775)

(1810-1816)

(1821-1835)*

901.011 Pères Récollets

*(1760-1796)

901.012 Gabriel-Jean Brassier, p.s.s., vic. gén.

(1789-1796)

901.013 Notice biographique de Mgr Plessis Érection du diocèse de Montréal

901,104 Mgr Lartigue -

de Mgr de Philadelphie

(1822-1823)

de Mgr de New York

(1826-1839)

de Mgr de Bardstown

(1821-1829)

de Mgr Poynter

901.015 Mgr Lartigue - Contre

l'admission des notables

(1831)

901.016 Mgr Lartigue -

Lettres personnelles

$(1821-1835)^{*}$

901.017 Messieurs McGuire et Tabeau -

Division de Québec et Biens

de Saint-Sulpice

901.018 Mgr Lartigue - Gouvernement anglais et Evêché de Mtl

(1826-1835)*

(1825-1835) *

901.019 Saint-Sulpice et Mgr Lartigue

901.020 Mgr Lartigue - Cathédrale (Comité, Plans, Commission des Travaux, Assurances)

(1821-1835)

(1822-1828)

901.021 Mgr Lartigue -

Questions des Notables

Bills pour communautés rel.

Erection des paroisses

Éducation

Écoles Normales

(1825-1831)

901.022 Mgr Lartigue -

Lettres de sa famille

(1788-1835)* 
901.023 Mgr Lartigue -

Recensement

Lettres de D.B. et J. Viger

re: écoles; autres documents

J. Viger et Lennox

901.024 Mgr Lartigue - Lettres de

M. François Bonin, p.s.s.

901.025 Messieurs J. G. Rocque et J. V.

Quiblier, p.s.s. à Mgrs Lar-

tigue et Bourget

$(1820-1835) *$

(1820-1834)

$(1826-1835) *$

901.026 Mgr Lartigue -

Lettres de plusieurs prêtres

901.027 Mgr Lartigue - Lettres de

M. Augustin Vervais, maître d'école

901.028 Mgr Lartigue -

(sans date)

(1811-1833)

Leilres de Mí. Múñitgólfier

Lettres de prêtres et d'amis

Règlements divers

Documents

*(1760-1820)

901.029 Lettres de M. Viau, vic. gén.

de M. Terrasse, prêtre

de M. Duclaux, prêtre

de M. Roux, p.s.s.

(1819-1833)

901.030 Pierre-Antoine Tabeau -

Ses troubles et ses hésitations

901.031 Voyages de P.-A. Tabeau dans le

Haut-Missouri

901.032 Lettres de Mgr Turgeon à M.

Marcoux, Sault-Saint-Louis

901.033 Mgr Lartigue -

Testament

M. F. J. Deguise (testament)

(1834-1835)

(1803-1805)

(1828-1835)*

$(1831-1835) *$

901.035 Requête des congréganistes de

Montréal au Gouverneur

901.036 Mgr Lartigue - Journal de

Voyage en Europe

(1803)

901.037 Mgr Lartigue - travaux

(1819-1820) 
re: Notes diverses adressées à un curé de campagne

Grammaire anglaise

(1830)

Libertés gallicanes

Philosophisme

901.038 Mgr Lartigue - divers, indulgences, règlements pieux, associations pieuses

(1823-1828)

901.039 Mgr Lartigue - Sermons

901.040 Mgr Lartigue -

Cas réservés dans le Québec

Coutumes de Paris Mandements de Québec

(1805-1822)

(1824)

901.041 Mgr Lartigue - Travaux d'Écriture Sainte

(sans date)

901.044 Diverses lettres à Mgr Bourget

901.045 Mgr Bourget -

Diverses nominations

Affaires personnelles

Testament

(1826-1836) *

901.047 Mgr Lartigue - Sermons

901.048 Mgr Lartigue - Correspondance

du Dr L. C. Blyth

(1789-1835)*

(sans date)

901.050 Jean-Baptiste Thavenet, p.s.s. à

$(1822-1835)^{*}$

Mgr Lartigue

901.052 Compagnie de la Baie d'Hudson

(1819-1826)

901.053 Mgr Bourget -

Lettres personnelles

901.085 Mgr Bourget -

M. Pinsonneault

M. Hicks

901.092 Education - Écoles Normales

(1820-1835)*

901.095 Autographes intéressants

(1826-1835)*

901.104 Affaires des sauvages

(1823-1835)*

901.114 Lettres de M. Roux, vic. gén.

$(1835)^{*}$

901.115 Lettres de M. Montgolfier, p.s.s.

(1796-1829)

901.116 Lettres à M. Cazeau, sec. de l'évêque de Québec

(1776-1789)

(1826-1835) 
901.136 Notre-Dame -

$$
\text { Division de la paroisse }
$$

*(1760-1835)*

901.137 Notre-Dame et Saint-Sulpice

*(1760-1835)*

901.138 Notre-Dame -

Copies de lettres de Mgrs Lartigue et Bourget réponses

901.140 Notre-Dame - Bâtisse de l'église $(1827-1835)^{*}$ (1816-1826)

901.146 Mandements - Fête des Saints (1777-1830)

901.148 Règlements au sujet des Registres,

Cas réservés, etc. . .

* (1727-1760)*

901.149 Succession J. M. K. Lennox

901.150 A Mgr Lartigue de D. B. Viger

(à suivre)

FrançoIs Bgaudin, prôtre 